\title{
A review of methods for estimating mortality due to parasites in wild fish populations
}

\author{
R. J. G. Lester \\ Department of Parasitology, University of Queensland; Brisbane, Australia 4067
}

\begin{abstract}
Six methods are described for detecting mortality due to parasitic infections in natural fish populations. They are: (a) through autopsies; (b) by determining the frequency of infections known to be eventually lethal; (c) by observing a decrease in the prevalence of a long-lived parasite (or permanent scar from a parasite) with host age; (d) by observing a decrease in the variance/mean ratio for the parasites with host age; (e) by comparing the observed frequency of a combination of two independent events with the calculated probability of their occurrence; and finally (f) by comparing the observed frequency distribution of the parasite, with a projected frequency based on data from lightly infected fish. In this technique, negative binomials are fitted to the data and truncated at various points. Some advantages and disadvantages of the different methods are given, together with examples. The methods do not necessarily provide definitive answers, but they are indicative of whether or not significant parasite-related mortality may be occurring, and in some cases provide an estimate of its probable magnitude in terms of the total host mortality rate.
\end{abstract}

\section{INTRODUCTION}

The role of parasitic disease in the ecology of natural populations of animals is not well understood. In theory, parasitic disease has the potential to be a major contributor to host mortality rates and even to regulate host population size. Its theoretical importance has been recognised for many years and has been restated recently by Anderson (1979, 1982) and Anderson \& May (1979, 1981).

In practice the importance of parasitic infections in host population biology is equivocal (Holmes, 1982). We can look at histological sections and predict the extent of the damage in individual fish; we can measure physiological changes in fish as a consequence of infection; we can even observe fish death in the laboratory as a result of an infection; but we cannot be sure of the relevance of these observations in the field.

Several approaches have been used in recent years to determine whether a parasitic infection is likely to be affecting the host mortality rate in a natural population. Other techniques need to be developed and all need to be more widely applied to give us some answers to what is a fundamental question in ecological parasitology. Six methods are described below together with an example of each. This is not an exhaustive list. Other techniques have been used in specific situations. For example, Burrough \& Kennedy (1979) found that stunting in roach as a result of high population density was alleviated following an epidemic of Ligula intestinalis. Many infected fish apparently died and this 
relieved the pressure of over-population that was affecting the roach, leading to an increased growth rate the following year among the survivors. Another method not discussed below but one which shows particular promise is the comparison of recovery rates of tagged diseased and healthy fish (Munro et al., 1983). As far as I am aware this has not yet been used.

No method by itself can provide a definitive answer. In most cases an observed positive result can arise from conditions other than increased host mortality. In each case the alternative explanations need to be ruled out before we can be reasonably sure we are monitoring parasite-associated losses. This usually requires further work such as additional sampling, or laboratory experiments. Kennedy (1983) has cautioned that even if there is a correlation between fish death and parasite burden it should not necessarily be taken as evidence of cause and effect. Correlated but independent events may be causing the change. Taking all these reservations into account, the methods described below, particularly the statistical methods, can be extremely useful for, at the very least, they can tell us which host-parasite systems we should look at more closely.

The methods when correctly interpreted can indicate whether fish carrying a particular parasite load are likely to have a higher mortality rate than less heavily infected or uninfected fish. Whether this is of significance to the fish population as a whole is a separate question that is not specifically dealt with here. It was addressed by Munro at this symposium in connection with Ichthyophonus in plaice (see Sindermann, 1984), and is considered in general terms in the section "Discussion".

\section{METHODS USED FOR ESTIMATING MORTALITY}

\section{Method 1: Through autopsies}

In human and veterinary medicine the main means of determining the cause of death is through a post-mortem examination. Though this may seem ideal, its findings may be misleading. Consider the case of a person who breaks a leg and as a result of his inactivity catches pneumonia from which he dies. Determining the factor that sets in motion a chain of events that result in death is difficult even when autopsies are possible. However, one rarely finds dead or moribund fish. This is presumably because predators and scavengers take sick fish before they die. Thus, if we were able to complete autopsies on all dead fish, the immediate cause of death in most species is likely to be "being eaten"! A further disadvantage of this method is that autopsies usually do not provide us with much idea of the proportion of the population affected, at least in wild populations.

Dead fish are usually seen during epizootics when large numbers are dying at one time. Annual mass mortalities of the mullet Liza carinata have been repeatedly observed in postspawning aggregations in a lagoon off the Gulf of Suez (Paperna \& Diamant, 1984). Large numbers of the monogenean Benedenia monticelli were found on dying and dead fish. The severe dermal erosion produced by heavy infestations of this parasite was thought to be a major contributor to the cause of death in these fish. Burreson (1981) found that 39 of $\mathbf{4 8}$ juvenile summer flounders trawled in late January from the lower York River, U.S.A., were dead. All fish from which blood could still be collected were infected with Trypanoplasma bullocki and most of the fish had severe ascites. The 
deaths coincided with a heavy mortality in experimental fish that had earlier been inoculated with $T$. bullocki. There were no deaths in the control (uninfected) group. He suggested that all yearling summer flounder that remained in Lower Chesapeake Bay during January 1981 died as a result of $T$. bullock $i$ infections.

If parasite-affected fish are normally taken by predators before they die, autopsies on predators can give us the proportion of parasitized versus non-parasitized fish being consumed. Dobben (1952), for example, found that cormorants in the Netherlands took roach parasitized by Ligula intestinalis more frequently than they took non-parasitized roach. The predation rate on the infected fish was about five times higher. To determine the significance of this differential mortality some measure of the overall mortality rate due to cormorants is needed.

\section{Method 2: Determining the frequency of lethal infections}

Though dead fish are rarely found, fish carrying infections that under laboratory conditions are invariably terminal are occasionally encountered. From the frequency of infection an estimate of the numbers of fish dying from the disease can be made. An obvious difficulty lies in the assumption that the laboratory results are directly applicable to the field. Also, as individual fish will vary in their resistance to the disease, it may be difficult to decide which infections in the field are going to lead to death, and over what time scale. A further complication is that death of larval or juvenile fish may occur in such a short time that few parasitized juveniles are ever caught.

Burreson (1984), in an extension of his work mentioned above, successfully applied this technique to Trypanoplasma-infected flounder caught along the Atlantic coast of North America. He estimated that 10 to $20 \%$ of the flounder off the coast of Virginia died each winter.

The fungus Ichthyophonus hoferi readily causes death in several marine and freshwater species in the laboratory and is likely to have the same effect in the field. Periodic epizootics off the North American east coast were believed to cause widespread mortality in herring and may have had a role in determining herring abundance (Sindermann, 1970). McVicar (1981) looked at infections in plaice in Scottish waters. He considered that the disease was invariably terminal, and estimated that $55 \%$ of the annual mortality in plaice in one area north of Scotland was attributable to the parasite. He went on to determine the proportion of infected fish producing detectable antibody to the parasite. Using this, together with information on the rate of antibody production and the form of the survivorship curve for infected fish, it is possible to calculate the average survival time for an infected fish, assuming rates of infection and antibody production are constant.

\section{Method 3: Observing a decrease in the prevalence of a long-lived parasite with host age}

Many parasites, particularly larval helminths, are thought to remain alive in the fish for extended periods, often for the life of the fish. Other parasites, though they die, leave a scar or a mass of amorphous necrotic tissue indicating their former presence. As a host cohort ages, the frequency of such parasites or scars will initially increase, and then after 


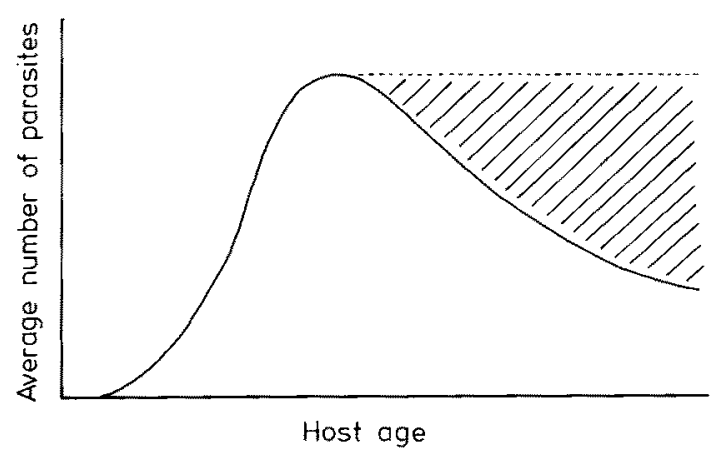

Fig. 1. Hypothetical example of a fish population in which the abundance of a long-lived parasite decreases with host age. The solid line represents the average number of parasites observed (or some other measure of degree of parasitaemia); the shaded area, the change in parasite abundance due to lost hosts

reaching a peak may decrease in the fish remaining alive (Fig. 1). If this happens, one possibility is that infected hosts are being selectively removed from the population sampled. (Other possibilities include poor sampling technique, and an erroneous assumption that the parasite or scar remains visible for life.)

It is best if one cohort can be followed throughout its life span or at least for a substantial period of its life (the "prospective" approach of Doll \& Hill, 1954), as the bulk of the infection may be picked up at one age and the availability of infective stages may vary from year to year. Unfortunately this can not often be done with any degree of certainty as the movements of natural fish populations are generally poorly understood. Thus, this potentially powerful technique, or any method that depends on sequential sampling, may be difficult to apply in many instances. In addition, for the method to work, heavily infected individuals have to survive long enough to appear in the peak sample.

Henricson (1977) found that the plerocercoids of both Diphyllobothrium dendriticum and $D$. ditremum in two year classes of char Salvelinus alpinus increased during the first seven and eight years, and then decreased in 8 and $9+$ fish. He concluded that the lower intensity of parasites in older char was caused by the death of heavily infected fish. (He did not determine what proportion were lost but as both parasites had overdispersed distributions the absence of just one or two heavily infected fish from his samples could have accounted for his results.)

Probably the best aquatic example of this technique, though not from a fish, is that of Perrin \& Powers (1980). They counted the lesions caused by the nematode Crassicauda sp. in the skulls of dolphins Delphinus delphis of different ages. Presence or absence rather than the number of parasites was used in their calculations, making the latter independent of the form of the parasite's distribution. They estimated that 11 to $14 \%$ of the total annual natural mortality was related to the parasitic infection.

Estimates based on this method are likely to be underestimates for if heavily infected individuals are disappearing from the population then the observed peak in the level of parasitaemia is likely to be too low. Perrin \& Powers had to assume that there was no mortality prior to the peak, and no new lesions occurred after the peak. 
Method 4: Observing a decrease in the variance/mean ratio for the parasites with host age

Gordon \& Rau (1982) proposed an ingenious method for detecting mortality associated with high levels of parasitaemia (Fig. 2). In their system, the fish Culaea inconstans were continually acquiring metacercariae of Apatemon gracilis. The mean number of

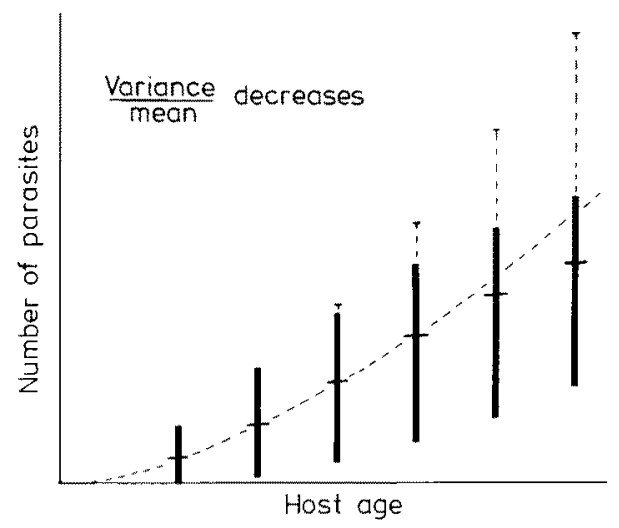

Fig. 2. Hypothetical example of a parasitized fish population in which the degree of dispersion of the parasite decreases with respect to the mean number of parasites, due to the loss of heavily infected individuals (Method of Gordon \& Rau). The vertical bars represent the dispersion observed; the horizontal bars, the observed means; the dotted curve connects the "true" means, i.e. if no mortality had occurred; the dotted vertical lines the "true" dispersion if no mortality had occurred

parasites per fish, the variance and the variance/mean ratio all increased with time, until at a certain point the variance stopped increasing and even began to decrease, thus causing a decrease in the variance/mean ratio. They concluded that the decrease was due to heavily infected fish being removed from the population.

In a subsequent paper, Anderson \& Gordon (1982) used simulation models to explore the theoretical aspects of this technique. Essentially their results confirmed its validity, but in addition they showed that chance effects become critically important when host sample sizes are small, and they pointed out that a decline in the degree of parasite dispersion in the older age classes may be generated by factors other than parasiteinduced mortality. The same effect could be caused by a reduction in the degree of heterogeneity in the infection rate of older fish, or by acquired immunity. There was no evidence that either of these was acting in the Culaea/Apatemon system. Gordon \& Rau (1982) did not try to estimate what proportion of the fish population was being removed. It may be easiest to do using a simulation model. Like the previous method, this method requires sequential sampling and thus is subject to the hazards that that entails.

Method 5: Comparison of the observed frequency of a combination of two independent events with the calculated probability of their occurrence

In a few cases parasitic infection may occur independently in two or more different organs. Consider a simple instance of this where only two organs are involved but the probability of the organs being infected is not necessarily the same. By the multiplicative 
law for generating the probabilities of combinations of independent events, the probability of both organs being infected is the product of the probabilities of each one being infected irrespective of the condition of the other. Suppose we are able to assess these probabilities; for example of left and right organs becoming infected; the product of these should give us some idea of the expectation of finding a fish with both sides infected, if all fish have the same risk of natural mortality. If mortality is greatest in the most heavily infected fish a smaller proportion of fish with both sides infected will be observed than predicted from the above calculation.

To calculate the probabilities of infection we cannot use the totals, as they are to some extent dependent on the number of double infections observed. Instead, they are calculated using a $2 \times 2$ table, right-infected and right-uninfected on one axis and leftinfected and left-uninfected on the other axis.

For example, flounder Atherestes stomias off British Columbia are occasionally parasitized by the copepod Phrixocephalus cincinnatus. This develops in the eye of the fish. Of a sample of 64 fish, Kabata (1969) found that 50 had the right eye infected, and 18 the left eye. Of these, 15 were double infections. From the $2 \times 2$ table above and assuming no fish die with single infections, the probability of getting an infection in the right eye was $35 / 46=0.76$, and in the left eye $3 / 14=0.21$. Thus, the probability of having both eyes infected was $0.76 \times 0.21=0.16$, i.e. 10 fish. This is less than the actual number observed with double infections, so the data suggest that there is no mortality associated with the infection.

This calculation is based on a relatively small number of fish. To carry out a formal test to determine whether the observed data are consistent with the hypotheses that fish survival is independent of infection, and left and right eye infections occur independently of one another, $\chi^{2}$ values can be calculated from the $2 \times 2$ table described above. In this example, the sum of the chi-squared values is 0.4 indicating that the observed and expected values are not significantly different. In fact, with a sample of only 64 fish, there would have to be a mortality of over $50 \%$ in the double eye infections before it could be detected at the $95 \%$ level of confidence.

Other observations on $P$. cincinnatus suggest that the parasite is quite pathogenic; fish with both eyes parasitized eventually become blind and probably die from starvation (Kabata, 1970). The failure of the statistical analysis to detect any increase in mortality perhaps reflects the small size of the sample (Fisher's Exact Test may help here), or an erroneous assumption that the left and right eye infections are independent events. It would be valuable to collect more data on this interesting parasite.

Method 6: Comparison of the observed frequency of the parasite with a projected frequency based on data from the lightly-infected fish

In most cases infection is not a random process and we have to resort to other means to predict the frequency of multiple infections. This requires making an assumption about the form of the parasite's distribution among the hosts at the time of infection. Parasite distributions are almost invariably overdispersed and usually follow a negative binomial type of distribution. This is the case considered here, though in some circumstances a different distribution may be more appropriate, such as a Poisson or Neyman A, in which case this would be used in place of the negative binomial. 
The method, first proposed by Crofton (1971), requires that mortality increases with the number of parasites present. Thus, the observed frequency distribution of the parasite (Fig. 3; histogram) will lack fish particularly in the tail of the distribution. In reality we do not know the values for the original distribution (represented by dots in the

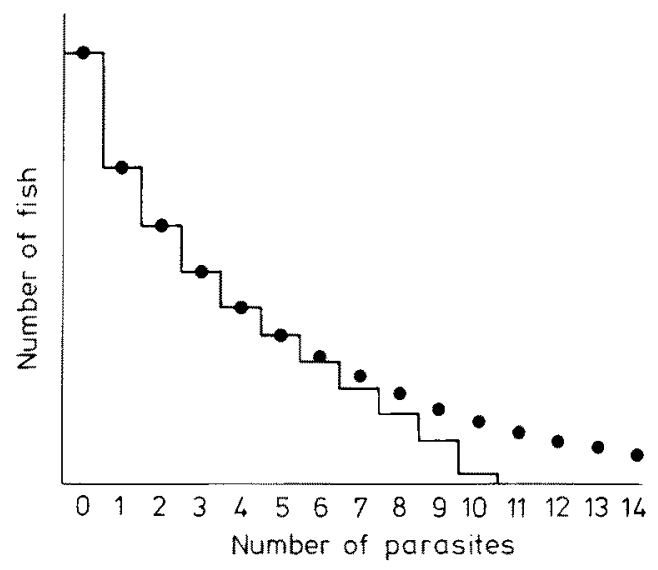

Fig. 3. Hypothetical example of the frequency distribution of a parasite in a fish population. The circles represent the original distribution at the time of infection, and the histogram the distribution observed after some of the more heavily infected fish had died. Theoretical distributions fitted to the observed data truncated at progressively fewer parasites will approach the original distribution

figure). However, we make the assumption that like the majority of parasites it followed some form of negative binomial reasonably closely. Values can be estimated for the negative binomial parameters $p, k$, and $N$ (the total number in the sample) by fitting the distribution to the observed data by one statistical means or another. The extent to which the distribution fits the data can be assessed by a $\chi^{2}$ statistic.

If we now omit the last observed point in the figure, and fit a negative binomial distribution to the frequencies of fish with 0 to 9 parasites, and subsequently 0 to 8,0 to 7 , etc, an increasing estimated value of $N$ will suggest that there are fewer heavily infected fish than one would expect from the frequency distribution of the lightly infected fish. The individual expected frequencies can be calculated for direct comparison with observed frequencies.

The method used by the author is an iterative technique, minimizing $\chi^{2}$ to determine the best fit (Lester, 1977). Local minima in the $\chi^{2}$ values can occur, so it is best to start with the full distribution and work back, truncating in small steps.

An unexpectedly infrequent occurrence of heavily infected fish in the sample may be the result of several factors other than increased host mortality, such as sampling error, development of avoidance behaviour or resistance in the infected fish, the loss of parasites from heavily infected fish, or choosing an inappropriate theoretical distribution. For the technique to work, small numbers of parasites should have little effect on mortality. In addition, the sample should either be drawn from fish of the same age, or from age classes after infection and mortality have occurred.

Adjei (pers. comm.) counted the numbers of Callitetrarhynchus gracilis blastocysts in 452 male and 445 female Saurida tumbil. In both sexes, as the fitted curve was 
progressively truncated, the estimated total number in the sample increased. In the males, a distribution fitted to the frequencies of fish with 0 to 3 parasites (Fig. 4) predicted that one would have expected about $5 \%$ more fish in the sample than was observed. From other observations it seemed likely that this was a result of parasite-

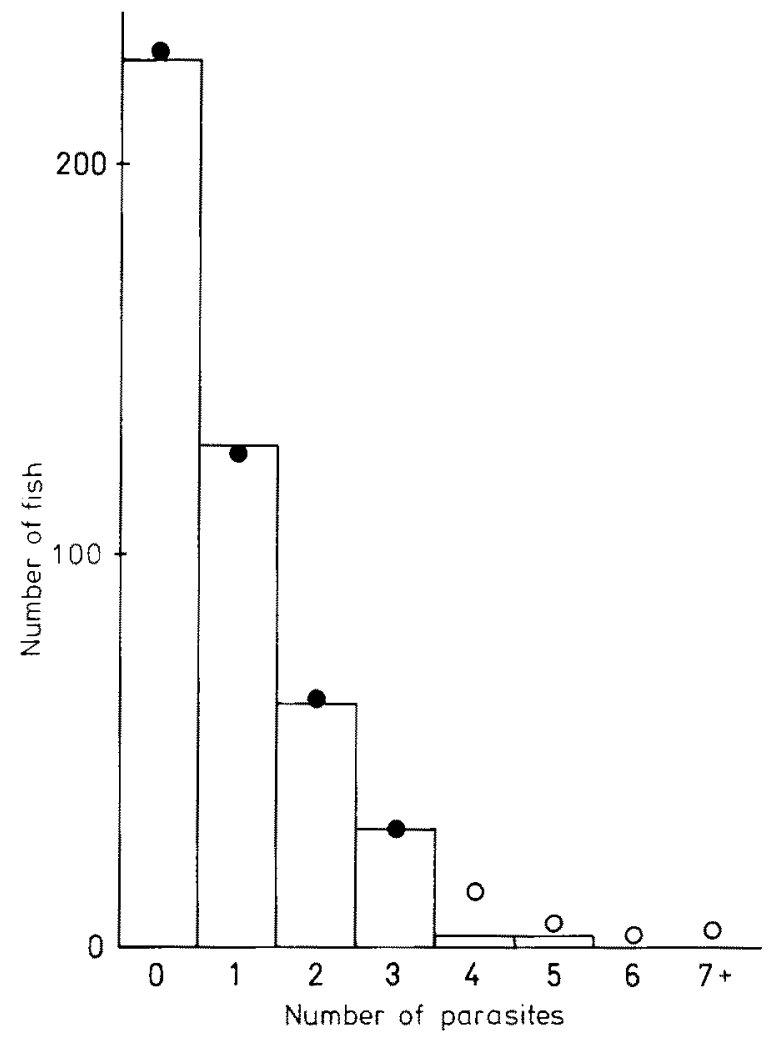

Fig. 4. The histogram represents the frequency distribution of blastocysts of Callitetrarhynchus gracilis in 452 male Saurida tumbil (from Adjei, pers. comm.). The circles are from the negative binomial distribution that best fits the first four points of the histogram

related mortality affecting the heavily-infected fish. In the females, the fitted curve predicted 2 to $3 \%$ more fish (Fig. 5).

However, the shapes of the curves were very different. Though the ratio of uninfected fish to fish with one parasite was similar in both sexes, relatively fewer fish with 2 or more parasites were observed in males than in females. This suggested that though the males and females were picking up parasites at much the same rate, multiple infections in the males were much rarer. Thus the third and fourth points of the male curve were probably not a reliable indicator of the original distribution.

Further evidence that the males were more affected by the parasite than the females comes from the results of the progressive truncations (Fig. 6). As the males were truncated, the biggest increase in the estimated total number occurred between 3 and 4 parasites per fish. In the females it was between 4 and 5 parasites per fish. Evidently four parasites per fish were more harmful to males than to females. 


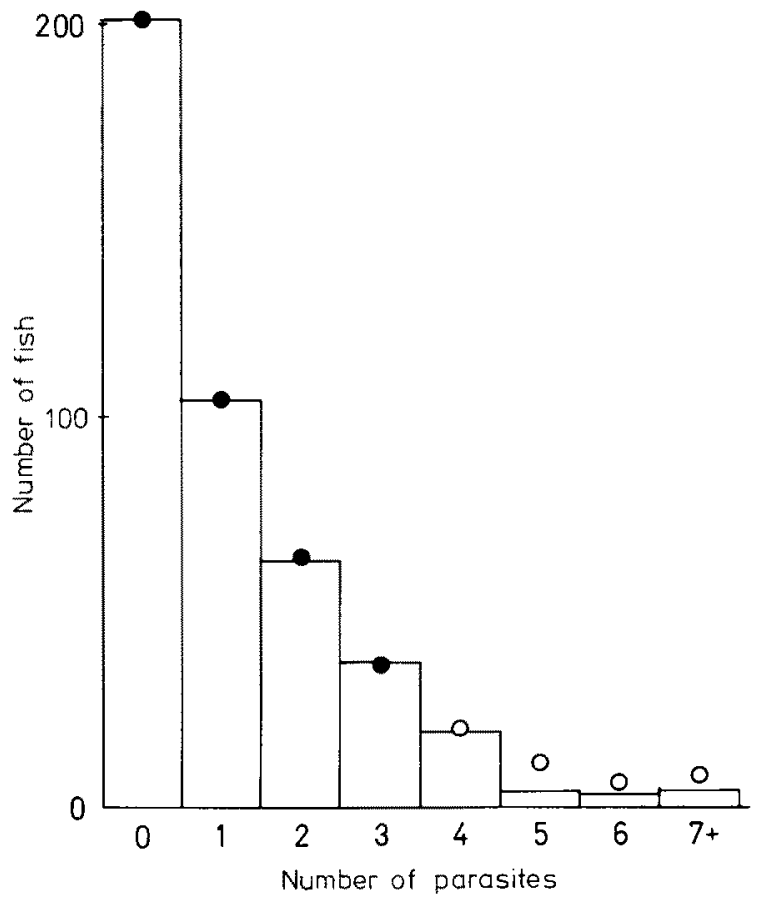

Fig. 5. Frequency distributions of $C$. gracilis blastocysts in 445 females $S$. tumbil. The circles represent the negative binomial distribution that best fits the first four values

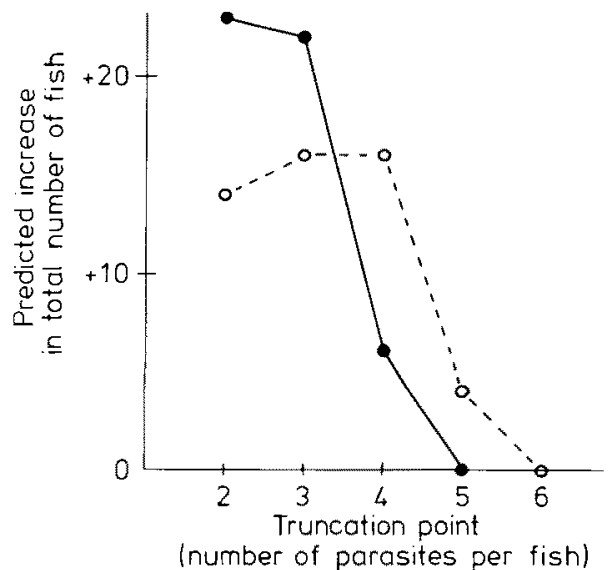

Fig. 6. The differences between the observed total number of fish and the estimated total number from distributions fitted to progressively more of the histograms in Figures 5 and 6 . Solid lines, male fish; dotted lines, female fish

As even the first few points of the male distribution were suspect, how could the shape of the original distribution be determined? Fortunately, they had the data from the females. The latter were caught at the same time, had evidently become infected at 
about the same size (100 to $140 \mathrm{~mm}$ ), when feeding on the same food (Adjei, pers. comm.). Their distribution, particularly when truncated at 3 parasites per fish, thus provided an estimate of the expected parasite distribution in male fish in the absence of parasite-related mortality. Figure 7 shows the results of the simplest application of this data: the predicted original numbers of males based on a negative binomial derived

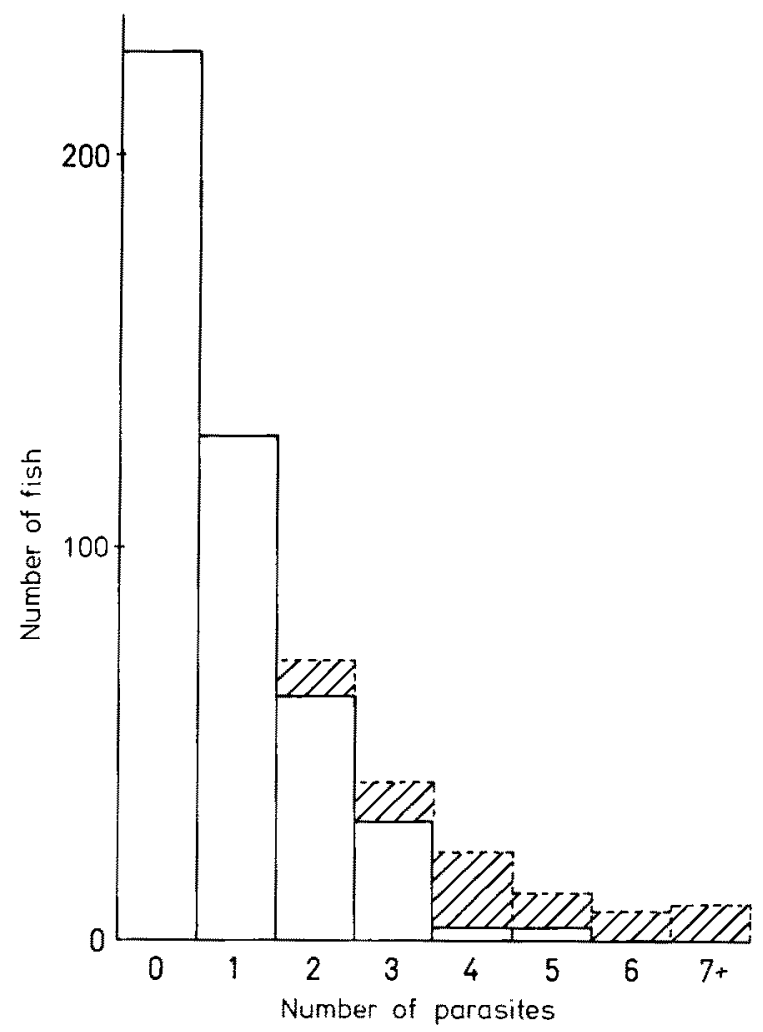

Fig. 7. Frequency distribution of the parasite in male fish (from Fig. 4) with an estimate of the probable original distribution (shaded area)

from the number of males with zero, and the $p$ and $k$ parameters estimated from the females. It predicts that about $12 \%$ of the males are missing from the sample. This is a minimum estimate as we have assumed that females with low numbers of parasites are not affected.

A more sophisticated statistical analysis of this data is in preparation.

\section{DISCUSSION}

I have attempted to show in this paper that there are ways we can detect the component of natural mortality that is associated with parasitic disease in a wild fish population. Animals die from a combination of factors, their position in time and place being perhaps as important as their physiological condition. Perhaps the question we 
should ask is, if a parasite increased the likelihood of death, is this of any consequence to the host population? When it occurs in relatively old postreproductive animals, the answer in general may be no, except if it allows more of a younger age group to survive. Mortality in pre-breeding individuals, however, may be much more important.

In practical terms, we need to tackle the question, if the parasite was absent, would there be more fish? In many cases the higher mortality rate of parasitized fish compared to non-parasitized may eventually be shown to be because they are selectively removed by predators (see for example Dobben, 1952). Predators take them presumably because it requires less energy to catch them compared to non-parasitized fish. If a predator has a fixed total amount of energy for catching prey, then if the parasite were absent, the predator would take less prey, and therefore one might expect that more prey would survive.

However, the factors that control population size are varied and frequently not well understood. Highly territorial species may be restricted by the availability of territory, others by the availability of shelter. In these cases, if the habitat is near its carrying capacity a decrease in losses associated with parasites may not increase total population size (though it may affect the age structure). On the other hand, where the size of a year class is more or less determined by say the availability of food during the early larval stages, then any additional mortality that occurs after that stage will presumably affect the population size. From a fisheries point of view., the period when disease-associated mortality is having its greatest effect may be, as Munro et al. (1983) have pointed out, after the fry stage and before the fish are recruited to the fishery. This is a stage which should receive more attention.

Where there is evidence, perhaps from one of the methods above, that an infection is causing serious mortality among adult fish, it may be possible to compare the age structure of two adjacent populations or sub-populations, one heavily parasitized and one more or less free of the parasite. In the parasitized population one would expect to find a greater proportion of younger individuals than in the non-parasitized population.

Finally, in theory, parasitic infections like other diseases, are able to regulate host population size. For true regulation to occur, losses associated with parasites should increase when the population is large and decrease when the population is small. Does this happen in reality? Until we have more results we cannot say.

Acknowledgements. I am indebted to Mr. A. Barnes of the University of Queensland for statistical advice, and to Dr. G. Lauckner of the Biologische Anstalt Helgoland for critically reviewing the manuscript.

\section{LITERATURE CITED}

Anderson, R. M., 1979. The influence of parasitic infection on the dynamics of host population growth. In: Population dynamics. Ed. by R. M. Anderson, B. D. Turner \& L. R. Taylor. Blackwell, Oxford, 245-281.

Anderson, R. M., 1982. Host-parasite population biology. In: Parasites - their world and ours. Ed. by D. F. Mettrick \& S. S. Desser. Elsevier, Amsterdam, 303-312.

Anderson, R. M. \& Gordon, D. M., 1982. Processes influencing the distribution of parasite numbers within host populations with special emphasis on parasite-induced host mortalities. - Parasitology 85, 373-398,

Anderson, R. M. \& May, R. M., 1979. Population biology of infectious diseases. Part II. - Nature, Lond., 280, 455-461. 
Anderson, R. M. \& May, R. M., 1981. Population dynamics of microparasites and their invertebrate hosts. - Phil. Trans. R. Soc. (B) 291, 451-524.

Burreson, E., 1981. Effects of mortality caused by the hemoflagellate Trypanoplasma bullocki on summer flounder populations in the Middle Atlantic Bight. - C. M./ICES G 61, 1-6.

Burreson, E., 1984. Effect of temperature on Trypanoplasma induced mortality of juvenile summer flounder Paralichthys dentatus. - Helgoländer Meeresunters. 37, 343-352.

Burrough, R. J. \& Kennedy, C. R., 1979. The occurrence and natural alleviation of stunting in a population of roach, Rutilus rutilus (L.). - J. Fish Biol. 15, 93-110.

Crofton, H. D., 1971. A model of host-parasite relationships. - Parasitology 63, 343-364.

Dobben, W. H. van, 1952. The food of the cormorants in the Netherlands. - Ardea 40, 1-63.

Doll, R. \& Hill, A. B., 1954. The mortality of doctors in relation to their smoking habits. - Brit. med. J. $1,1451-1455$.

Gordon, D. M. \& Rau, M. R., 1982. Possible evidence for mortality induced by the parasite Apatemon gracilis in a population of brook sticklebacks (Culaea inconstans). - Parasitology 84, 41-47.

Henricson, J., 1977. The abundance and distribution of Diphyllobothrium dendriticum (Nitzsch) and $D$. ditremum (Creplin) in the char Salvelinus alpinus. - J. Fish Biol. 11, 231-248.

Holmes, J. C., 1982: Impact of infectious disease agents on the population growth and geographical distribution of animals. In: Population biology of infectious disease agents. Ed. by $R$. M. Anderson \& R. M. May. Springer, Berlin, 314 pp.

Kabata, Z., 1969. Phrixocephalus cincinnatus Wilson, 1908 (Copepoda, Lernaeoceridae): morphology, metamorphosis and host-parasite relationship. - J. Fish. Res. Bd Can. 26, 921-934.

Kabata, Z., 1970. Crustacea as enemies of fishes. T. F. H. Publ., Jersey City. 171 pp.

Kennedy, C. R., 1983. Problems of disease assessment in natural fish populations: a population ecologist's view of diplostomiasis. - Int. Symp. Ichthyoparasit. Czech. Acad. Sci. 1, 46-47 (Abstract).

Lester, R. J. G., 1977: An estimate of the mortality in a population of Perca flavescens owing to the trematode Dipolostomum adamsi. - Can. J. Zool. 55, 288-292.

McVicar, A. H., 1981. An assessment of Ichthyophonus disease as a component of natural mortality in plaice populations in Scottish waters. - C. M./ICES G 49, 1-8.

Munro, A. L. S., McVicar, A. H. \& Jones, R., 1983. The epidemiology of infectious disease in commercially important wild marine fìsh. - Rapp. P. v. Réun. Cons. int. Explor. Mer, 182, $21-32$.

Paperna, I. \& Diamant, A., 1984. Monogenean infections and mortality in wild and cultured Red Sea fishes, - Helgoländer Meeresunters. 37, 445-462.

Perrin, W. F. \& Powers, J. E., 1980. Role of a nematode in natural mortality of spotted dolphins. $-J$. Wildl. Mgmt. 44, 960-963.

Sindermann, C. J., 1970. Principal diseases of marine fish and shellfish. Acad. Press, New York, 369 pp.

Sindermann, C. J., 1984. Report of informal session on disease in marine fish. - Helgoländer Meeresunters. 37, 633-639. 